\title{
THE ROLE OF IC TECHNOLOGIES ON BIOMEDICAL ULTRASONICS
}

\author{
Pai-Chi Li \\ Department of Electrical Engineering \\ National Taiwan University, Taipei, Taiwan 106, ROC
}

\begin{abstract}
In this paper, we will discuss the role of modern IC technologies on ultrasonic medical imaging. A digital phased array system for ultrasonic imaging is first described and design principles of receive beamformation are reviewed. Evolution of the beamformer design and state of electronics will also be related. The paper then describes three emerging imaging technologies, and discusses the challenges in circuit design for overcoming these challenges before potential of these techniques can be fully realized.
\end{abstract}

\section{INTRODUCTION}

The capabilities of ultrasonic imaging systems have been closely linked to state-of-the-art electronic technologies [1]. In the early applications of ultrasound in medical imaging, B-scans were generated using mechanical scanning of a single element transducer and the image is displayed by varying the intensity of an appropriately scanned CRT beam using analog circuits [2]. In the late 1970s, digital scan converters began to replace analog converters with more efficient data interpolation. In the $1980 \mathrm{~s}$, micro-processors were widely used in programmable imaging systems such that optimal imaging parameters can be used given a particular clinical application. Also in the late 1970s and early 1980 s, phased array systems were made possible by advances in analog delay lines and the reduced size of analog processing elements. At that time, typical systems were limited to fixed-focus receive operations because the noise introduced by changing delay line taps adversely affected image quality. In 1979, nonetheless, a method for dynamic delays without changing delay taps by heterodyning the radio frequency signal to an intermediate frequency was patented [3]. In the $1990 \mathrm{~s}$, digital processing was pushed completely into the front-end of a phased array system, thanks to the advances in analog-to-digital (A/D) conversion led by many IC manufacturers. As the electronic technologies continue to advance according to the Moore's law, many high-speed computational tasks now become feasible and higher performance and more functions are now possible. These new imaging capabilities include real-time three-dimensional imaging, volumetric imaging using two-dimensional atrays, harmonic imaging and spatial compounding. In this paper, we will first describe the general architecture of an ultrasonic imaging system, and then we will focus on the role of the IC technologies on the evolution of the beamformer, which is a critical component in a high-end phased array system. Finally, three emerging imaging techniques will be introduced and the associated challenges will be described.

\section{SYSTEM ARCHITECTURE}

Figure 1 shows a schematic diagram of the receiver of a digital, phased array ultrasonic imaging system. A transducer array connects to the system via a cable. On the transmit side (not shown in this figure), the beamformer determines the delay pattern and pulse waveform that set the desired transmit focal point. The outputs of the beamformer are then amplified and drive the transducers. Signals received by the transducer array are first amplified in order to compensate for the depth dependent signal loss during propagation. This is also known as time gain compensation (implemented by a low noise amplifier and $a$ variable gain amplifier). The amplified signals are then digitized and sent to the receive beamformer for dynamic receive focusing. The beamforming can be implemented in analog or digital form, but it is mostly digital in modern systems except for continuous wave Doppler processing whose dynamic range is mostly too large to be processed through the same channel as the image.

The pre-detection filter following the receive beam former serves the purpose of pulse shaping (axial filtering), beam shaping (lateral filtering) and temporal filtering (filtering between image frames). In other words, it is used to alter the frequency contents (both temporal and spatial) of the received signals. Additionally, it can be used for data re-sampling (interpolation or decimation). Typically; only the envelope of the received signal is displayed. The envelope detector is used to filter out the carrier frequency component and to keep only the magnitude of the remaining signal. The envelope detected signal is also called the video signal. The post-detection filter is applied to the detected signal for the purpose of smoothing, edge enhancement or a combination of both. It is typically used to enhance contrast or spatial resolution such that particular features in the image can be better perceived. Additional data re-sampling can also be performed at this stage. Other processing, such as re-mapping (gray scale or color) and digital

0-7803-8665-5/04/\$20.00 @2004 IEEE 
gain adjustment, can be applied following the post-detection filter.

Scan conversion is the process of converting the acquired ultrasound data (e.g., polar coordinate data for a sector scan format) into the Cartesian raster data used by standard display monitors. In order not to introduce artificial artifacts, such as a Moire pattern, two-dimensional interpolation is usually involved for most pixels in the image and hence it is computationally demanding. Ideally, the imaging parameters in all the aforementioned functional blocks should be controlled and adjusted adaptively. In other words, the system should be optimized automatically based on clinical needs, patient characteristics and users" personal preference. However, due to implementation difficulties, most systerns are not fully adaptive and the optimization is usually done manually.

\section{BEAMFORMER ARCHITECTURES}

The receive beamformer hardware implements a "delay-and-sun" process and it is relatively complicated due to the need for dynamic focusing. Figure 2 shows a simple model for a digital receive beamformer. Note that each array element has its own delay value and the value is time (depth) varying. To ensure bigh quality beam formation, the delay accuracy $\Delta \tau$ must be chosen such that $\Delta \tau \leq 1 / 32 f_{0}$, where $f_{0}$ is the carrier frequency of the ultrasonic waves. Most commercial ultrasonic imaging systems have frequencies ranging from $1.5 \mathrm{MHz}$ to $15 \mathrm{MHz}$. At $10 \mathrm{MHz}$, for example, the above equation indicates that $\Delta \tau$ needs to be less than 3.1ns. In a digital system, this means that the clock frequency needs to be as high as $320 \mathrm{MHz}$. Even if the frequency of the A/D converter only needs to satisfy Nyquist criterion, the beamformer still requires a much higher sampling frequency for high focusing quality (i.e., the $A / D$ converter output needs to be up-sampled to a bigher rate). At the current technological stage, this is very difficult (i.e., expensive) to achieve. This is the main reason why the interpolator is necessary.

An alternative way to achieve high focusing accuracy at relatively low clock frequencies in a digital system is to perform beam formation in baseband [4]. In other words, the original radio frequency signals are demodulated down to baseband before delays are applied. Using the previous criterion, the delay quantization interval $\Delta \tau$ only needs to satisfy the criterion $\Delta \tau \leq 1 / 32 \Delta f$, where $\Delta f$ is the difference between the true carrier frequency and the demodulation frequency. Since $\Delta f$ can be made much smaller than $f_{0}, \Delta t$ can be much coarser (i.e., cheaper) than that in a radio frequency beam former. Although the delays are much cheaper, there is still a phase term that needs to be taken care of as a result of complex demodulation. Generally, this is done very accurately by simply rotating the phase of the baseband signal using processors such as CORDIC. A simple block diagram of a baseband beam former is shown in Figure 3. Coarse time delays as well as accurate phase rotations on baseband signals are indeed an altemative way of interpolation. Digital baseband beam formers provide high quality focusing at a lower cost by trading accuracy in time delays for complex signal processing.

With the rapid advancement of electronic technologies, alternative beam formation architectures have become feasible. One example is the Delta-Sigma beam former which takes advantage of high clock rate [5]-[6]. Conventional digital beamformers use $8-10$ bit A/D converters at clock frequencies between $20-40 \mathrm{MHz}$. Delta-Sigma converters, on the other hand, typically use 1 bit conversion at a very fast clock rate. Note that if the clock rate is 32 to 64 times the carrier frequency of the sound waves, delay architecture can be significantly simplified. In addition, 1 bit conversion may also dramatically reduce the size and cost of a digital beamformer. A block diagram of the Delta-Sigma based beamformer is shown in Figure 4. From the evolution of an ultrasonic beamformer, the role of IC technologies on the beamformer design and the resultant image quality improvement is clear.

\section{CHALLENGES}

\subsection{Analog Doppler beamformer: dynamic range requirements}

Although the widespread use of digital beamformation has proven its advantages over conventional analog beamformation, a dedicated Doppler beamformer is still employed in several high-end ultrasonic imaging systems due to its need for a high dynamic range and signal-to-noise ratio. As a result, improved sensitivity and clarity of blood flow information throughout the entire spectral waveform can be achieved. If a single digital beamformer is to be used for all function modes, approximately at least a 15 bit SNR A/D converter with a sampling rate higher than $40 \mathrm{MHz}$ will be needed.

\subsection{Real-time volumetric imaging: requirements for computational power}

Dynamic receive focusing in ultrasonic array imaging involves extensive real-time computations and data communication. Particularly for three-dimensional imaging using fully-sampled two-dimensional arrays, implementation of dynamic focusing can be extremely complicated because of the large channel count. We have proposed an efficient dynamic focus control scheme for a delay-and-sum based 
beamformer [7]. The scheme simplifies dynamic focus control by exploiting the range dependent characteristics of the focusing delay. Specifically, the overall delay is divided into a range independent steering term and a range dependent focusing term. Since the focusing term is inversely proportional to range, approximation can be made to significantly simplify dynamic focus control at the price of minimal degradation in focusing quality at shallow depths. In addition, the aperture growth can be utilized to devise a non-uniform quantization scheme for the focusing delay values. Efficacy of the proposed scheme has been demonstrated. One example shows that with the proposed dynamic focus control scheme, a 4096 element array only requires 227 independent controllers for the range dependent focusing term. Moreover, only 28 non-uniform quantization levels are required to achieve the same focusing quality as that of a conventional scheme with 784 uniform quantization levels.

Although efficacy of this efficient beamforming scheme has been demonstrated, approximation was made and the image quality trade-offs exist in certain circumstances. In addition, the mid- and back-end signal processing is also very computationally demanding in order to meet the real-time imaging criterion. Therefore, the computation power provided by future electronic technologies will be critical in making real-time three-dimensional volumetric imaging practical.

4.3. Aperture domain processing: requirements for higher data transfer bandwidth

The aperture domain processing includes a class of new imaging methods [8]. It is based on the data from individual channels before they are summed in the beamformer. In other words, it utilizes the raw radio frequency data and has been applied to areas such as adaptive imaging, parallel beamformation and blood flow estimation. The architecture is schematically shown in Figure 5. Take adaptive imaging as an example, it can be used to derive an image quality index known as the generalized coherence factor (GCF).

The GCF is derived from the spatial spectrum of the received aperture data after proper receive delays have been applied. It is defined as the ratio of the spectral cnergy within a prespecified low-frequency range to the total energy. It is demonstrated that the low-frequency component of the spectrum corresponds to the coherent portion of the received data, and that the high-frequency component corresponds to the incoherent portion. In addition, the GCF is also an index of the focusing quality and can be used as a weighting factor for the reconstructed image. In the presence of phase aberrations, which result in image quality degradation, the GCF based adaptive imaging technique outperforms the other techniques and has shown its capabilities in reducing image artifacts. However, the main challenge is in the data transfer bandwidth, because all the channel data, instead of just the beam sum, need to be transferred and buffered for further signal processing.

\section{CONCLUSIONS}

In this paper, evolution of a digital phased array system for clinical ultrasonic imaging has been reviewed, with an emphasis on the beamformer design. In addition, some of the emerging imaging technologies were described, and the associated challenges were discussed. With the state of electronics continues to be driven by Moore's law in the next few years, it is expected that the clinical values of ultrasound will continue to increase.

\section{ACKNOWLEDGMENTS}

The author would like to thank J.J. Huang, H.-L. Liu and M.-L. Li for the research work related to this paper.

\section{REFERENCES}

[1] M. O'Donnell, "Applications of VLSI circuits to medical imaging," Proc. IEEE, vol. 76, pp. $1106\{1114$, Sep. 1988.

[2] C. F. Schueler, H. Lee, and G. Wade, "Fundamentals of digital ultrasonic imaging," IEEE Trans. Sonics Ultrason., vol. SU-31, pp. 195 \{217, July 1984.

[3] S. H. Maslak, "Acoustic imaging apparatus," U.S. Patent 4,140,022, February 20, 1979.

[4] M. O'Donnell, W. E. Engeler, J. T. Pedicone, A. M. Itani, S. E. Noujaim, R. J. Dunki-Jacobs, W. M. Leue, C. L. Chalek, L. S. Smith, J. E. Piel, R. L. Harris, K. B Welles, and W. L. Hinrichs, "Real-time phased array imaging using digital beam forming and autonomous channel control," Proc. IEEE Ultrason. Symp., 1990, pp. 1495-1498.

[5] S. R. Freeman, M. K. Quick, M. A. Morin, R C. Anderson, C. S. Desilets, T. E. Linnenbrink, and $M$. o' Donnell, "Delta-sigma oversampled ultrasound beamformer with dynamic delays," IEEE Tran. Ultrason., Ferroelect., Freq. Contr., vol. 46, tno. 2, pp. 320 - 332, Mar. 1999.

[6] P.-C. Li, J-J Huang, H.-L. Liu and M. O'Donnell, "A dynamic focusing technique for delta-sigma based beamformers", Ultrasonic Imaging, vol. 22, pp. 197-205, October, 2000.

[7] P.-C. Li and J.-J. Huang, "Efficient dynamic focus control for three-dimensional imaging using two-dimensional arrays" IEEE Transactions on Ultrasonics, Ferroelectrics and Frequency Control, Vol. 49, No. 9, pp. 1191-1202, September, 2002.

[8] P.-C. $\mathrm{Li}$ and M.-L. Li, "Adaptive imaging using the generalized coherence factor", IEEE Transactions on Ultrasonics, Ferroelectrics and Frequency Control, Vol. 50, No. 2, pp. 128-141, February, 2003. 


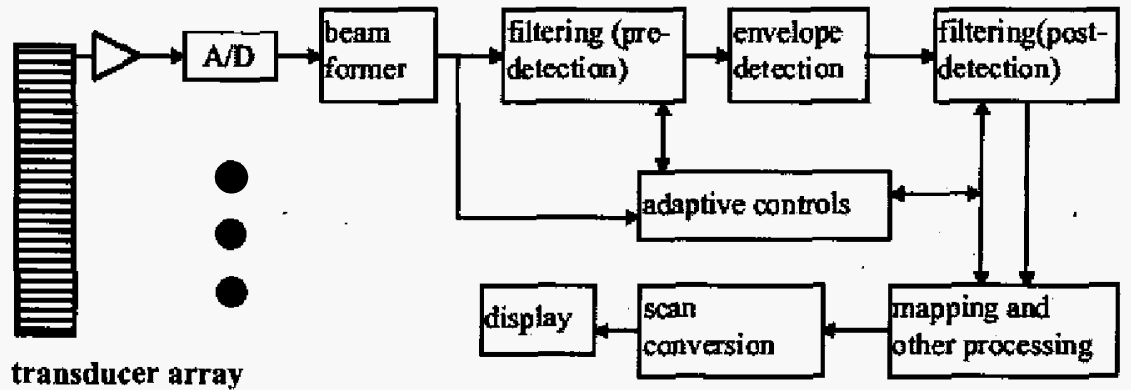

Figure 1: Schematic diagram of the receiver a digital phased array system for ultrasonic imaging



Figure 2: A digital receive beamformer using the radio frequency acoustic data

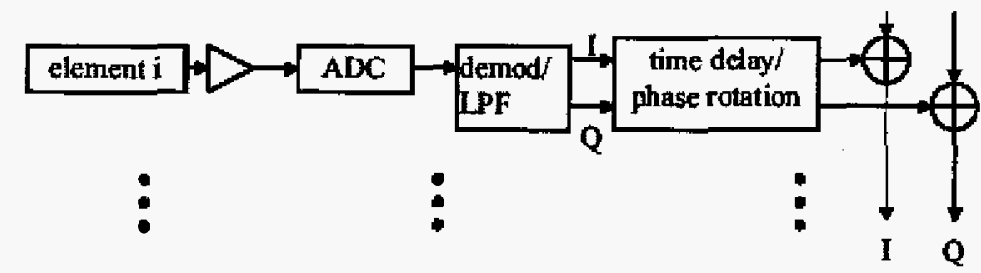

Figure 3: A baseband receive beamformer



Figure 4: Schematic diagram of a beamformer using Delta-Sigma modulators



Figure 5: Illustration of the aperture domain adaptive imaging technique 